\title{
Evaluation of Arterial Diameter by Mathematical Transformation of APG for Ambulatory Stiffness
}

\author{
Arathy $\mathrm{R}^{1}$, Nabeel P M${ }^{1}$, Jayaraj Joseph ${ }^{2}$, Abhidev V V², Mohanasankar Sivaprakasam¹,2 \\ ${ }^{1}$ Department of Electrical Engineering, Indian Institute of Technology (IIT) Madras, Chennai, India \\ ${ }^{2}$ Healthcare Technology Innovation Centre (HTIC), IIT Madras, Chennai, India
}

\begin{abstract}
Non-invasive, continuous measurement of arterial stiffness indices has established utility in cardiovascular risk stratification. This study aims to develop a subjectspecific model of soft tissue sandwich from the common carotid artery wall to the skin surface layer using acceleration plethysmograph (APG) waveforms. It was then used to estimate the lumen arterial diameter waveform using APG for stiffness evaluation. The carotid $A P G$ waveforms were collected using the developed accelerometer probe and its associated measurement system. The relationship between carotid diameter and $A P G$ from the neck was evaluated via mathematical models using system identification in MATLAB. The performance of the developed model for non-invasive assessment of carotid diameter and stiffness indices was validated on 15 subjects.

The developed model was implemented in real-time and continuously evaluated carotid diameter using APG from the neck. An RMSE of less than $0.14 \mathrm{~mm}$ was observed between the constructed carotid diameter waveform (using $A P G)$ when compared with an actual diameter measured using the ultrasound-based system. The study results demonstrated the feasibility of a subject-specific skintissue model with APG waveforms for arterial diameter measurement and estimation of the vessel stiffness.
\end{abstract}

\section{Introduction}

Arterial wall mechanics plays a crucial role in blood circulation throughout the arterial tree. It has been researched for its clinical implications of cardiovascular and vascular diseases. Arterial wall radial motion has been investigated for the estimation of the wall stiffness, neglecting the longitudinal movement [1]. The arterial wall stiffness evaluation helps in the early diagnosis of atherosclerotic or arteriosclerotis changes in the arterial tree for preventing the development of vascular diseases. Indirect measures of arterial stiffness include the assessment of arterial compliance, arterial distensibility, Young's modulus, and pulse wave velocity [2]. Ultrasound imaging is a well-known non-invasive technique for direct visualization and motion estimation of the arterial vessels. But these state-of-art devices demands sophisticated instrumentation and trained operators. Hence are not suitable for long term monitoring [2].

We present a method and instrumentation for measuring the arterial stiffness. The study initially shows a theoretical viscoelastic model of soft tissue sandwiched from the carotid arterial wall to the skin surface layer. Further, a black-box model was derived, simulations were performed based on the acquired set of single APG waveform input and diameter waveform output data and processed using MATLAB System Identification toolbox. This identified model along with the input surface APG waveform can be used to obtain the actual carotid lumen diameter waveform for arterial stiffness evaluation.

\section{Theoretical modelling}

The carotid arterial wall is found to be viscoelastic [3]. The wall elasticity is related to the elastin content, whereas the viscosity is related to the collagen and smooth muscle content. The arterial wall anatomy is composed of three layers, namely intima, media, and adventitia separated by elastic membranes. The arterial luminal surface, which is in contact with the blood, is lined with a layer of endothelial cells. The shear stress developed by the blood flow is applied to the endothelial cells. Among the above mentioned three layers of the arterial wall, the media supports the load induced by the blood pressure, and hence its mechanical properties represent the properties of the arterial wall [3]. The carotid arterial wall surface is surrounded by geometrically and mechanically nonlinear

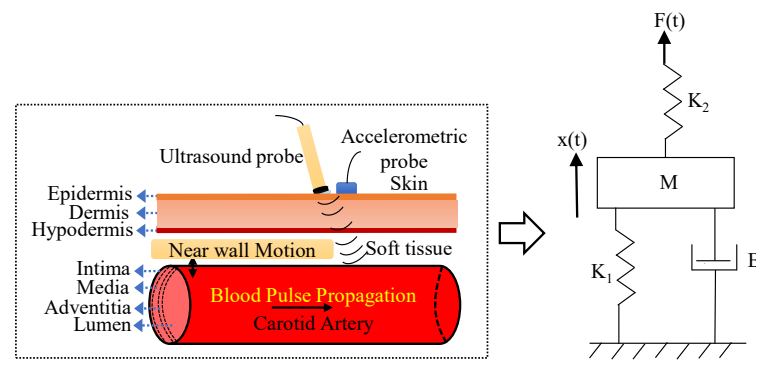

Figure 1. (a) Schematic representation of probe placement above the carotid arterial wall and its surrounding soft tissue layers. (b) Equivalent dynamic system model. 
biological soft tissues such as muscle, and skin [4-6]. The skin consists of three layers epidermis, dermis, and hypodermis. But the contribution of the fat layer and epidermis to the mechanical response of the skin are negligible. It is assumed that the dermis is the unique element responsible for the skin mechanic [7].

Considering the above properties of the different layers, and due to the time-harmonic nature of the radial motion of the arterial wall, the wall and its surrounding soft tissues are modelled as a linear system with displacement $\mathrm{x}(\mathrm{t})$ and axial blood flow force $F(t)$. It is impossible to propose a perfect mathematical model for the real carotid arterial wall and soft tissues. But as in initial attempt, a linear model was assumed that could approximate linear dynamic behaviour neglecting the nonlinear properties. The proposed theoretical model (Fig. 1) consists of the arterial wall and soft tissue system in series. The arterial wall can be modelled as a spring $\left(\mathrm{K}_{1}\right)$ and dashpot $(\mathrm{B})$ in parallel as in Fig. 1. The skin and the other soft tissues system respond differently under applied pressure as compared with the load cell [7]. These soft tissues reaction can be represented as a linear spring-mass system with spring constant $\left(\mathrm{K}_{2}\right)$ as in Fig. 1. The transfer function for this proposed system is:

$$
\frac{\mathrm{X}(\mathrm{s})}{\mathrm{F}(\mathrm{s})}=\frac{1}{\left[\mathrm{Ms}^{2}+\mathrm{Bs}+\left(\mathrm{K}_{1}-\mathrm{K}_{2}\right)\right]}
$$

With mass $M=1$, the driving force $F(s)$, the damping coefficient $\mathrm{B}$, the spring stiffness $\mathrm{K}_{1}$, and $\mathrm{K}_{2}$. Previous studies [2] have shown that the carotid surface APG waveform displays a significant similarity with the axial blood flow force pattern through the carotid artery. Also, it was found that this axial blood flow is responsible for the radial motion of the arterial wall. Due to these two results, the APG waveform from the carotid surface can be used to approximate the driving force $\mathrm{F}(\mathrm{t})$. Therefore in the present in-vivo study, APG waveform is taken as the input to the system to estimate the carotid lumen diameter waveform.

\section{Materials and methods}

\subsection{In-vivo experimental setup}

The overall instrumentation setup developed for the experimental validation of the proposed accelerometric system for continuous carotid lumen diameter measurement and stiffness estimation is depicted in Fig. 2. The carotid APG waveform was collected using an accelerometric patch probe (LIS344ALH, STMicroelectronics) and its associated measurement system with a sampling frequency of $1 \mathrm{kHz}$. This designed patch probe derives the APG waveforms associated with the displacement of the carotid vessel due to the influx of blood pulse wave through the carotid artery. Also, as shown in Fig. 2, our clinically validated image-free ultrasound technology ARTSENS $^{\circledR}$ device [9] was integrated with the accelerometric system for developing subject-specific mathematical modelling using the realtime acquired APG and diameter waveform. This model is used for reconstructing the lumen diameter waveform using the accelerometer system alone, and the ARTSENS ${ }^{\circledR}$ is used as the reference for the validation study. The reference device consists of a probe with a single-element ultrasound transducer (centre-frequency $=5 \mathrm{MHz}$ ) to acquire A-scan ultrasound frames for continuous measurement of lumen diameter. The device analog frontend consists of a pulser-receiver circuitry for operating the ultrasound transducer in pulse-echo mode and procuring A-scan at high frame rates using ARTSENS ${ }^{\circledR}$ technology and its automated software architecture for continuous evaluation of diameter [9].

The separation distance between the accelerometric probe and the single element ultrasound transducer is confirmed to be very minimal for a single site measurement of both the waveforms as illustrated in Fig. 2. The simultaneous acquisition of these waveforms with a sampling rate of $1 \mathrm{kHz}$ was achieved by synchronizing the analog front-end of the accelerometric system with ARTSENS $^{\circledR}$ device hardware. Further, the systolic $\left(\mathrm{P}_{\mathrm{s}}\right)$ and diastolic BP's $\left(\mathrm{P}_{\mathrm{d}}\right)$ are measured using an upper arm

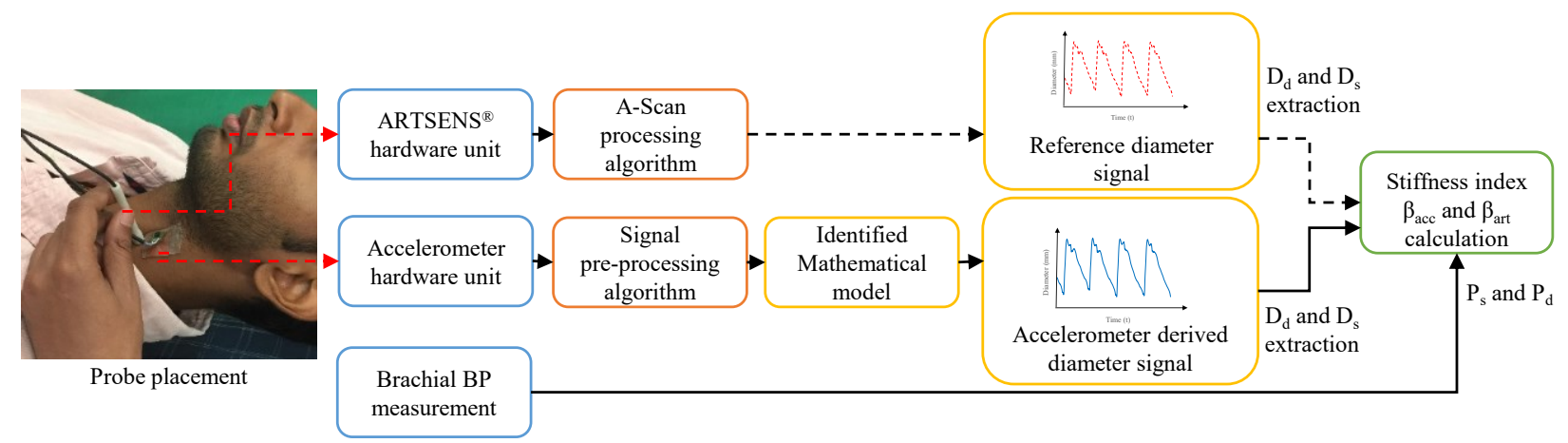

Figure 2. Developed measurement system for carotid lumen diameter and arterial stiffness estimation. 


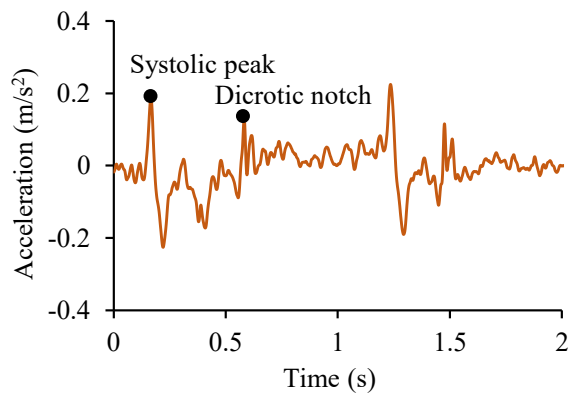

Figure 3. Continuous carotid skin surface APG waveform from a volunteer.

clinical-grade sphygmomanometer $\left(\operatorname{SunTech}{ }^{\circledR} 247^{\mathrm{TM}}\right)$ to obtain the related carotid $\mathrm{P}_{\mathrm{s}}$ and $\mathrm{P}_{\mathrm{d}}$ values [9]. The relationship between the carotid lumen diameter and surface APG from the neck was evaluated via the mathematical model estimated and validated using the System Identification performed in MATLAB software. This carotid arterial wall and surrounding soft tissues system model along with real-time acquired APG waveform results in carotid lumen diameter. Hence by the continuous acquisition of carotid arterial diameter and deriving the diastolic diameter $\left(\mathrm{D}_{\mathrm{d}}\right)$, and systolic diameter $\left(D_{s}\right)$ for each cardiac cycle with corresponding $P_{s}$ and $P_{d}$ measures, arterial stiffness index $\beta$ can be evaluated in a beat-by-beat manner as:

$$
\beta=\frac{\ln \left(\frac{\mathrm{P}_{\mathrm{s}}}{\mathrm{P}_{\mathrm{d}}}\right)}{\left(\frac{\mathrm{D}_{\mathrm{s}}}{\mathrm{D}_{\mathrm{d}}}-1\right)}
$$

\subsection{Data collection}

An in-vivo experimental study was conducted on a study population of 15 healthy subjects (mean age $=30 \pm$ 5 years, 10 males, and 5 females). The study protocol confirmed the Helsinki Declaration and informed consent was obtained from all the recruited participants. The main objective of the study was the evaluation of the carotid lumen diameter by mathematical transformation of the surface APG waveform for the estimation of stiffness and comparison with the reference method, to evaluate the accuracy of the proposed system.

All the experimental readings were performed by a single operator after asking the subjects to rest for 5 minutes in the supine position. The accelerometric and ultrasound probes were placed on the left side of the neck above the carotid artery for the subjects after identifying the exact location with palpation as in Fig. 2. It should be noted that two datasets were taken for each subject (D0, and D1), measuring approximately $15 \mathrm{sec}$ for model estimation and later $25 \mathrm{sec}$ for the validation study in MATLAB software. Each collected data set consist of a

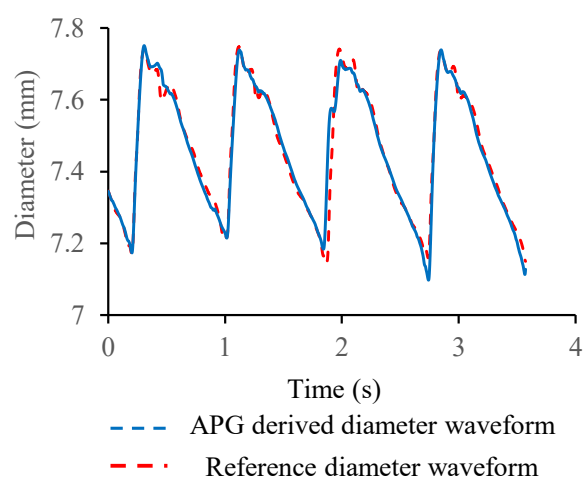

Figure 4. Overplot of lumen diameter measurement from an accelerometric system with ARTSENS ${ }^{\circledR}$ reference.

surface APG waveform, a carotid lumen diameter waveform, and brachial BP readings.

\subsection{System identification and validation}

The mathematical model was developed using a blackbox modelling approach. Initially, from the experimental data's, the APG waveform, which is related to the axial blood flow force, was regarded as the input $(\mathrm{u}(\mathrm{t}))$ to this unknown model. The carotid diameter waveform was considered as the corresponding output $(\mathrm{y}(\mathrm{t}))$ from the hidden model. Afterwards, multiple linear and nonlinear model structures are generated and chosen based on previous knowledge along with trial and error method. These selected models are then identified and validated on other data sets to see whether they meet the model requirements

\section{Experimental results and discussion}

The proposed accelerometric system yielded highly reliable continuous non-invasive APG waveform from all the subjects, as shown in Fig 3. Also, high fidelity lumen diameter waveform was simultaneously acquired with the above signals with ARTSENS ${ }^{\circledR}$ device for estimating the mathematical model in real-time and later as a reference for diameter and stiffness measurement validation. These recorded waveforms were found to hold relatively fewer phase delays due its time synchronized hardware acquisition, the matched frequency responses of the designed systems and very close separation distance between the two probes.

These real-time collected APG input and carotid diameter data were tested with linear models, and later with nonlinear models. To validate the authenticity of the model, the best fitting between the simulation response of the identified model and the actual diameter was recognized. Fundamentally, a model with best-fit percentage signifies how much that model can represent 


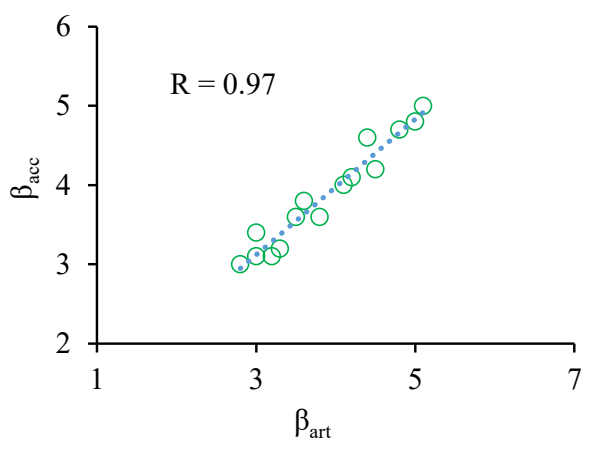

Figure 5. Regression plot of $\beta$ acc versus $\beta$ art.

the real carotid arterial wall and surrounding soft tissues system. Among this, the nonlinear autoregressive (ARX) model with exogeneous variable provided the best performance with $(1,2,1)$ model structure when compared with other models, including linear models. The accuracy of the model was examined by comparing the model simulated output with the reference device output in MATLAB. The fit percentage was more than $90 \%$ for all the subjects. Fig. 4. illustrates the fitting result of the model output with the reference diameter at the same time instant.. Similar results were obtained for all the recruited subjects with reasonable accuracy. The solid lines are the APG derived diameter waveform using the model, and the dashed line is the reference device diameter waveform. Apart from the best fit, autocorrelation of residuals and cross-correlation analysis were examined as well. The confidence level for both correlations was set at $95 \%$ (pvalue of 0.05 ) and was within the range.

A linear dynamic model was assumed in the theoretical modelling to describe the layers of the carotid arterial wall and its surrounding soft tissues for its computational efficiency. This model, however, does not consider the effect of the nonlinear properties. But human biological soft tissues shows the nonlinear properties and composed of inhomogeneous structures. Hence this blackbox modelling for real tissue yielding a nonlinear model provides a better model with good agreement. The rootmean-square-error less than $0.14 \mathrm{~mm}$ was observed between the simulated diameter waveform when compared with the exact diameter measured using the reference system at that time instant. Also, a beat-to-beat variation of less than $8 \%$ was observed for the estimated end-diastolic diameter $\left(D_{d}\right)$ and distension $(\Delta D)$. The correlation plot for the arterial stiffness index between the accelerometric system and reference was found to have $\mathrm{R}=0.97$ (as Fig.5). The carotid stiffness index demonstrated acceptable accuracy when compared with the reference system.

\section{Conclusion}

This work has proposed a method for mathematical transformation of the carotid surface APG waveform for the determination of the changes in lumen diameter of the carotid artery. The theoretical model-based analysis considered a linear system model of the arterial wall and its surrounding soft tissues to explain the dependency of the carotid APG waveform and the lumen diameter. A nonlinear ARX system model was obtained using system identification toolbox in MATLAB with the real-time acquired data. The feasibility and accuracy of the developed model were further validated using a new set of data for all the subjects. The study results demonstrated the possibility of using a subject-specific carotid arterial wall and surrounding soft tissues model with APG waveform as the input for arterial diameter measurement and hence for vessel stiffness evaluation.

\section{References}

[1] D. Wang et al., "Radial and longitudinal motion of the arterial wall: Their relation to pulsatile pressure and flow in the artery," Physical Review E, vol. 98, pp. 1-8, 2018.

[2] T. Pereira et al., "Novel methods for pulse wave velocity measurement," J. Med. Bio. Eng., vol. 35, no. 5, pp. 555-565, 2015.

[3] K. Hayashi, "Mechanical Properties of Soft Tissues and Arterial Walls", in Biomechanics of Soft Tissue in Cardiovascular Systems, Springer, vol. 441, 2003.

[4] K. Isogai, "Skin-fat-muscle urethane model for palpation for muscle disorders", proc. IEEE International Symposium on System Integration, Japan, 2015.

[5] A. Murai et al., "Dynamic skin deformation simulation using musculoskeletal model and soft tissue dynamics" Computational Visual Media, vol. 3 no.1, pp. 49-60, 2017.

[6] G. Lambert, "Mathematical and computational modelling of skin biophysics: a review", proc of The Royal Society A., vol. 473, no: 2203, pp. 1-38, 2017.

[7] John G. Webster, 'Biopotential electrodes", in Medical Instrumentation Application and Design, $3^{\text {rd }}$ edition, $\mathrm{pp}$. 196-200, 2014.

[8] J. Joseph et al., "Technical validation of ARTSENS-an image free device for evaluation of vascular stiffness", IEEE $J$. Transl. Eng. Heal. Med., vol. 3, pp. 1-13, 2015.

Address for correspondence:

Arathy R

Department of Electrical Engineering, Indian Institute of Technology Madras (IIT M), Chennai, India -600036

Email: arathyvysakh@gmail.com,ee14d017@ee.iitm.ac.in 